\section{Dysfonctions mitochondriales et vieillissement musculaire}

\section{Une mise à jour}

Julie Faitg ${ }^{1,2 *}$, Olivier Reynaud
Jean-Philippe Leduc-Gaudet
Gilles Gouspillou,
1,2,4

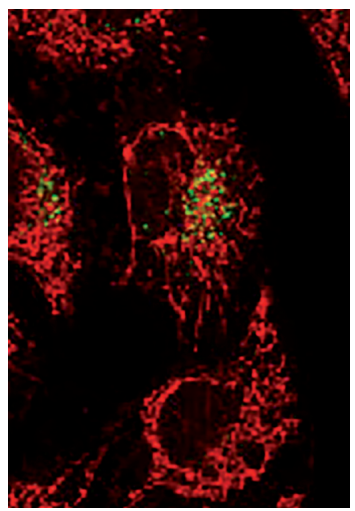

${ }^{1}$ Département des sciences de l'activité physique, faculté des sciences, Université du Québec à Montréal (UPAM), 141, avenue du Président Kennedy, H2X 1Y4 Montréal, Canada.

${ }^{2}$ Groupe de recherche en activité physique adaptée, Montréal, Canada.

${ }^{3}$ McGill University, Montréal, Canada.

${ }^{4}$ Centre de recherche de l'institut universitaire de gériatrie de Montréal, Montréal, Canada. *Ces auteurs ont contribué

manifeste par des pertes progressives de masse et de force musculaires. Ce processus biologique est nommé sarcopénie [1]. La sarcopénie est de manière égale au présent manuscrit.

gouspillou.gilles@uqam.ca lité, à l'augmentation du nombre de chutes et à l'état de fragilité des personnes âgées $[1,2]$, nuisant, de ce fait, gravement à la qualité de vie des personnes qui en sont affectées. Illustrant l'importance épidémiologique de ce phénomène, une étude conduite en 2002 aux États-Unis a estimé qu'approximativement $64 \%$ des hommes et $31 \%$ des femmes âgés de plus de 60 ans présentaient une masse musculaire en dessous de la gamme de valeurs normalement observée dans la population de jeunes adultes en bonne santé [2]. Cette étude a également estimé que le coût annuel de la sarcopénie, en 2002, s'élevait aux États-Unis, à plus de 18 milliards de dollars [2]. Étant donné l'augmentation constante de la proportion d'individus âgés de plus de 60 ans, qui devrait, selon les estimations de l'ONU (Organisation des Nations Unies), atteindre $22 \%$ de la population mondiale en 2050, l'identification des mécanismes impliqués dans le développement de la sarcopénie représente l'un des enjeux de santé publique majeurs que doivent affronter nos systèmes de recherche en santé.

L'étiologie de la sarcopénie est extrêmement complexe et multifactorielle. Elle représente une thématique de recherche actuellement très active. De multiples mécanismes biologiques ont été proposés pour expliquer son développement (voir [3] pour une revue détaillée). Parmi ceux-ci, l'accumulation au cours du vieillissement, de dysfonctions 
mitochondriales au sein des cellules musculaires, est un mécanisme qui a attiré énormément d'attention au cours des dernières décennies. La première partie de notre revue sera consacrée à une courte présentation des rôles et des fonctions que les mitochondries assurent au sein des cellules musculaires. Elle se poursuivra ensuite par une revue de l'état des connaissances sur le rôle joué par l'accumulation de dysfonctions mitochondriales dans le développement de la sarcopénie. Elle se conclura par une présentation des mécanismes potentiellement à l'origine de l'accumulation des dysfonctions mitochondriales observées au cours du vieillissement musculaire.

\section{Les multiples fonctions des mitochondries}

Les mitochondries sont des organites intracellulaires à double membrane qui proviennent de l'insertion symbiotique, il y a environ deux milliards d'années, d'une protéo-bactérie par un précurseur de la cellule eucaryote actuelle (voir [4] pour une revue détaillée). Cette insertion a abouti à la formation d'un organite présentant une membrane interne délimitant la matrice mitochondriale, et une membrane externe, ces deux membranes étant séparées par l'espace intermembranaire (Figure 1). Les mitochondries renferment un génome, le génome mitochondrial, qui est circulaire et localisé dans la matrice mitochondriale. II code 13 protéines qui sont toutes constituantes des complexes de la phosphorylation oxydative [4]. Cette phosphorylation oxydative, au travers de l'activité de la chaîne respiratoire (une chaîne constituée de 4 complexes protéiques numérotés de I à IV), est à l'origine de la création d'un gradient électrochimique de protons $\left(\mathrm{H}^{+}\right)$le long de la membrane interne de la mitochondrie. Ce gradient est utilisé par l'ATP synthase pour la production d'adénosine tri-phosphate (ATP), une molécule qui joue un rôle central dans le métabolisme énergétique cellulaire (Figure 1). Par leur rôle essentiel dans la production d'ATP, les mitochondries sont ainsi très souvent assimilées à des «usines cellulaires de production d'énergie».

La chaîne respiratoire, qui représente le site principal de consommation de l'oxygène dans l'organisme, est également un lieu important de production d'espèces activées de l'oxygène ( $E A 0$, ou ROS en anglais). Les complexes I et III de la chaine respiratoire peuvent ainsi générer I'anion superoxyde $\left(\mathrm{O}_{2}{ }^{-}\right)$, l'un des principaux membres de la famille des $\varepsilon A 0$.

L'un des autres rôles importants de la mitochondrie est sa capacité à réguler les flux calciques de la cellule. En effet, des transporteurs de calcium $\left(\mathrm{Ca}^{2+}\right)$, comme l'uniport calcique, qui permettent l'entrée des ions $\mathrm{Ca}^{2+}$ dans la matrice mitochondriale, et des échangeurs $\mathrm{Ca}^{2+} / \mathrm{H}^{+}$et $\mathrm{Ca}^{2+} / \mathrm{Na}^{+}$, permettant la sortie $\mathrm{du} \mathrm{Ca}^{2+}$ de la matrice mitochondriale, sont présents au sein des mitochondries. La relation étroite entre mitochondries et réticulum endoplasmique $(R E)$, réserve cellulaire calcique par excellence, permet à ces organites de jouer un rôle important dans l'homéostasie calcique cellulaire. Au niveau mitochondrial, le $\mathrm{Ca}^{2+}$ matriciel module l'activité de multiples déshydrogénases, dont la pyruvate déshydrogénase (PDH), et de l'ATP synthase, stimulant ainsi la production d'ATP. Cependant, une activation trop importante de ces déshydrogénases pourrait conduire à une production excessive de $\mathrm{NADH}, \mathrm{H}^{+}$qui pourrait, au travers de l'augmentation d'activité du complexe I qu'elle peut induire, augmenter le taux de réduction des pools de quinones et de cytochrome $C$, et, ainsi, favoriser la production d'EAO par la chaîne respiratoire [5]. L'entrée de calcium dans la mitochondrie dépend principalement du canal à anions dépendant du voltage (VDAC), qui permet aux ions de traverser la membrane externe mitochondriale, et de l'uniport calcique qui favorise la traversée de la membrane interne du calcium [6]. L'accumulation de calcium matriciel sera à l'origine de son interaction avec la Cyclophiline D, ce qui provoque l'ouverture du pore de transition de perméabilité (PTP). Celle-ci peut conduire à la rupture de l'intégrité des membranes mitochondriales et à la libération de facteurs pro-apoptotiques, normalement séquestrés dans les mitochondries [7]. L'augmentation de calcium matriciel provoque également un accroissement de la production d'EAO qui, eux aussi, peuvent déclencher l'ouverture du PTP [7]. L'ouverture du PTP est finalement à l'origine d'une baisse du potentiel membranaire mitochondrial conduisant à l'altération de l'énergétique mitochondriale [8].

Le PTP représente donc un passage au travers de la structure bi-membranaire de la mitochondrie. Sa composition moléculaire exacte reste encore inconnue. Néanmoins, son ouverture semble régulée par des interactions entre la Cyclophiline D, I'ATP synthase, I'ANT (adenine nucleotide translocase) et le transporteur de phosphate, quatre protéines de la membrane interne mitochondriale, avec VDAC (voltage-dependent anion channel), une protéine de la membrane externe de la mitochondrie [7-9]. La sortie du cytochrome $\mathrm{c}$ des mitochondries, comme d'autres facteurs pro-apoptotiques, comme APAF-1 (apoptotic protease activating factor-1) et l'endonucléase $G$, causée par l'ouverture du PTP, aboutit à l'activation de l'apoptose [7] et au clivage de l'ADN nucléaire de la cellule. Dans la plupart des cellules, le processus d'apoptose conduit à leur mort. Dans les cellules musculaires, il peut avoir des conséquences beaucoup plus complexes. Les cellules musculaires sont en effet plurinucléées et il est généralement admis que l'apoptose des cellules musculaires aboutit en fait, non à la mort de la cellule, mais à la perte de l'un ou de plusieurs noyaux [10] (Figure 2). La perte d'une fibre musculaire par apoptose nécessite donc une activation prolongée et généralisée des voies apoptotiques afin que l'entièreté des noyaux soit endommagée et que finalement la cellule meurt.

Les mitochondries, parce qu'elles jouent des rôles importants dans le métabolisme énergétique, la production de radicaux libres, l'homéostasie calcique et la régulation de l'apoptose, sont essentielles au fonctionnement et à la survie des cellules musculaires. 


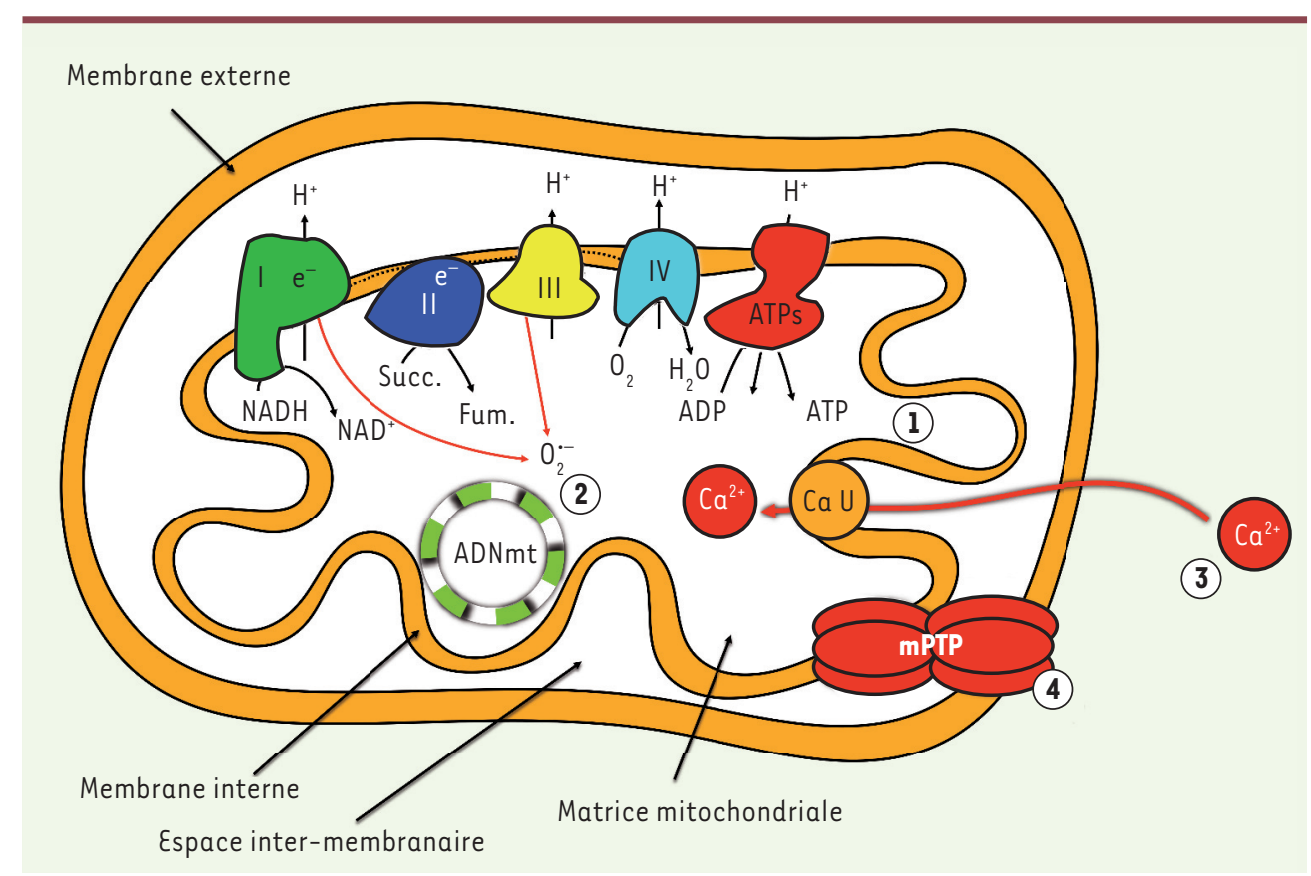

Figure 1. Les principales fonctions des mitochondries. Les mitochondries, au travers de l'activité de la chaîne respiratoire (composée des complexes I à IV, localisés dans la membrane interne) et de I'ATP synthase, permettent la synthèse d'ATP (1) ). Les mitochondries sont également une source importante d'anion super oxyde $\left(\mathrm{O}_{2} \bullet^{-}\right)$un radical libre dérivé de l'oxygène (2)). Grâce à leur haut potentiel de membrane et à la présence dans la membrane interne de l'uniport calcique, les mitochondries jouent un rôle important dans la régulation de l'homéostasie calcique (3) ). Les mitochondries, au travers du pore de transition de perméabilité (PTP), sont impliquées dans la régulation du déclenchement de I'apoptose (4)). I, II, III, IV : complexes protéiques de la chaîne respiratoire ; I : complexe I (NADH-coenzyme $Q$ oxydoréductase) ; II : complexe II (succinate-coenzyme $\rho$ oxydoréductase) ; III : complexe III (coenzyme Q-cytochrome C oxydoréductase) ; IV : complexe IV (cytochrome C oxydase) ; ATPs : ATP synthase ; mPTP : pore de transition de perméabilité mitochondrial ; ADNmt : ADN mitochondrial ; $\mathrm{CaU}$ : unipore calcique ; $\mathrm{O}_{2}{ }^{--}$: anion superoxyde ; Fum : fumarate ; NAD : nicotinamide adénine dinucléotide ; NADH : nicotinamide adénine dinucléotide réduit ; Succ : succinate.

\section{Mitochondries et vieillissement musculaire}

\section{Production mitochondriale d'espèces activées de l'oxygène et vieillissement musculaire}

Les mitochondries, au travers de leur fonctionnement normal, produisent des espèces activées de l'oxygène (EA0). Il est en effet généralement reconnu que les mitochondries sont la principale source d'EAO dans les cellules. Les $\varepsilon A 0$, si elles sont produites en excès, peuvent être à l'origine de dommages oxydatifs à l'encontre de nombreuses molécules de la cellule et de structures cytosoliques. Partant de ce constat, Harman a été le premier, en 1956, à émettre une théorie selon laquelle le vieillissement cellulaire pourrait avoir pour origine une accumulation de dommages moléculaires, à l'intérieur de la cellule, due à l'action oxydante des sous-produits du métabolisme oxydatif [11]. L'accumulation de dommages serait, selon sa théorie, à l'origine d'un dysfonctionnement cellulaire et causerait le vieillissement de la cellule [11]. Par la suite, Harman étoffa sa théorie en suggérant que les mitochondries, parce qu'elles sont les principales productrices d'EAO dans la cellule, seraient les responsables de cette action oxydante [12]. En lien avec cette théorie, de multiples études, réalisées par la suite, s'accordèrent sur le fait que le vieillissement musculaire était associé à une augmentation de marqueurs du stress oxydant [13-17]. Plusieurs études ont également rapporté une augmentation de la production d'EAO par les mitochondries musculaires au cours du vieillissement chez l'homme [18, 19], chez le rat [17] et chez la souris [20]. Renforçant la théorie de Harman, il a été récemment démontré que la surexpression, spécifiquement dans les mitochondries, de la catalase (une enzyme qui transforme le peroxyde d'hydrogène $\left[\mathrm{H}_{2} \mathrm{O}_{2}\right]$ en eau et en dioxygène), atténuait les effets du vieillissement sur la force musculaire chez la souris [21].

II est cependant important de noter ici que l'hypothèse d'une association entre augmentation de la production mitochondriale d'EAO et vieillissement est loin de faire l'unanimité. En effet, un nombre non-négligeable d'études n'a trouvé aucune augmentation de la production mitochondriale d'EAO au cours du vieillissement musculaire, que ce soit chez l'homme [22, 23] ou chez le rat [24]. Deux études récentes, qui se sont intéressées aux effets de la supplémentation en anti-oxydants ciblant spécifiquement les mitochondries (le Mitop - un mimétique du coenzyme $\left(10\right.$ - et le SS-31 ${ }^{1}$ ), mettent également à mal l'implication d'une surproduction d'EAO dans le développement de la sarcopénie [25, 26]. Dans ces 2 études, dans lesquelles les supplémentations en anti-oxydants ont été initiées avant l'apparition de la sarcopénie et maintenues pendant 15 semaines, aucun

${ }^{1}$ De formule : D-Arg-2', 6'-diméthyltyrosine-Lys-Phe- $\mathrm{NH}_{2}$. 


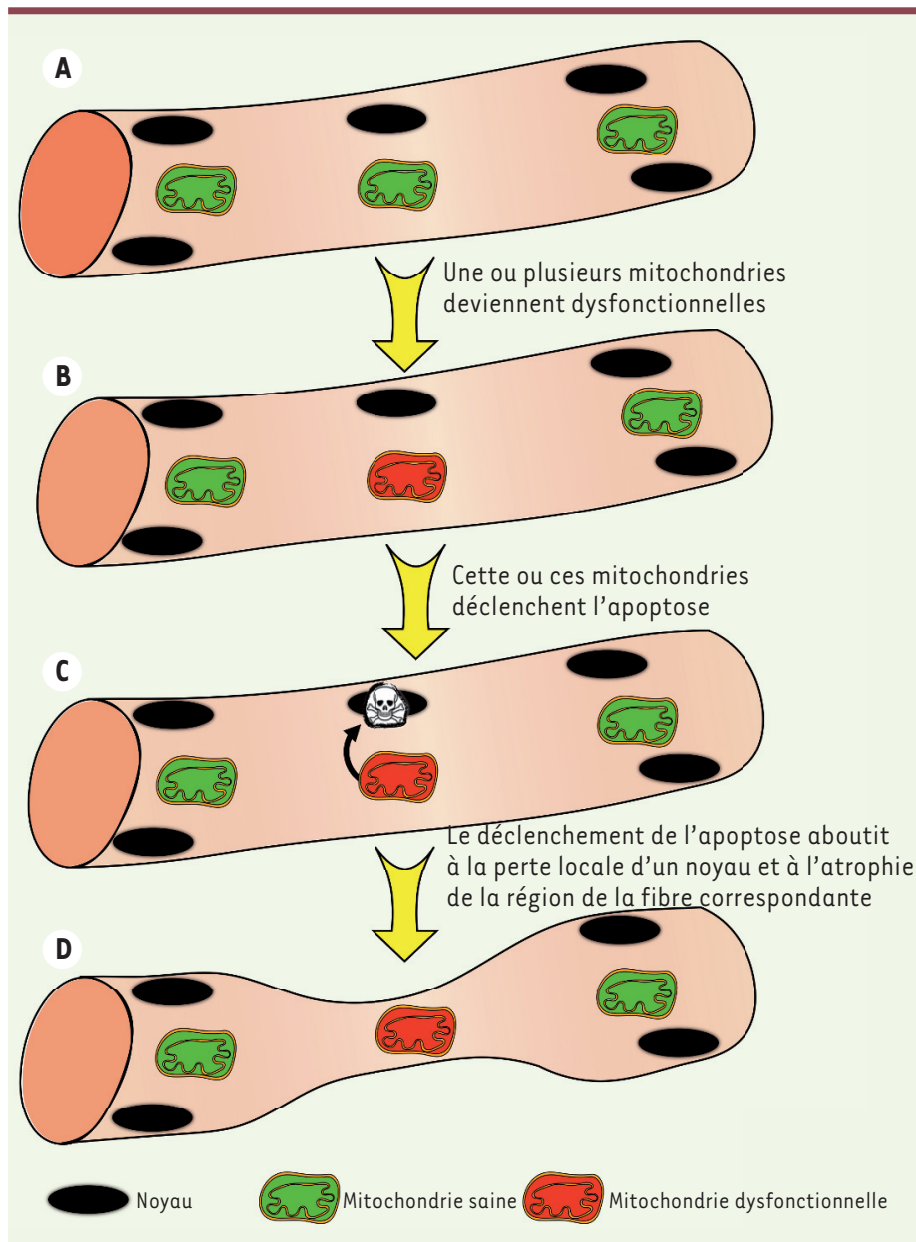

Figure 2. Impact du déclenchement de l'apoptose par les mitochondries dans les cellules musculaires. A. Représentation d'une fibre musculaire contenant des mitochondries saines. Une mitochondrie de cette fibre musculaire devient dysfonctionnelle (B) et déclenche le programme apoptotique qui aboutit à la perte du noyau à proximité $(c)$. La perte de ce matériel génétique réduit localement la capacité de synthèse protéique, ce qui cause une atrophie locale de la fibre musculaire (D).

effet protecteur contre la sacropénie n'a été observé, ce qui remet en cause l'importance des $\varepsilon A 0$ produits par les mitochondries dans le développement de la sarcopénie [25, 26].

Ainsi, bien que la plupart des études s'accordent sur le fait que le vieillissement musculaire est associé à l'apparition d'un stress oxydant, l'implication des mitochondries dans ce processus reste incertaine $[56](\rightarrow)$.

\section{$\rightarrow$ Voir la Synthèse de R. Barouki, $\mathrm{m} / \mathrm{s}$ $n^{\circ} 3$, mars 2006, page 266}

\section{Énergétique mitochondriale et vieillissement musculaire}

Bien que plusieurs études aient rapporté une absence de relation entre activité de la chaîne respiratoire mitochondriale et vieillissement musculaire $[22,23,27,28]$, un consensus semble cependant émerger, ces dernières années, sur le fait que l'énergétique mitochondriale est effectivement altérée au cours du vieillissement musculaire. Le vieillissement musculaire paraît en effet s'accompagner d'une réduction de la vitesse maximale de consommation d'oxygène, que ce soit chez l'homme [29-31], le rat [32-36], ou chez la souris $[20,37]$. Conley et al. ont ainsi observé que le fonctionnement intrinsèque des mitochondries (c'est-à-dire leur capacité de respiration maximale) était altéré avec le vieillissement musuclaire [31]. Cette observation a été remise en cause par d'autres équipes $[23,24]$. II a cependant été mis en évidence, et cela de manière unanime, que la capacité maximale de production d'ATP par les mitochondries musculaires était réduite chez l'homme âgé [38], mais aussi chez le rat $[34-36,39,40]$ et la souris [41]. Une étude récente a également pu mettre en évidence, in vivo chez le rat, une altération de la réponse des mitochondries à une augmentation de la demande en ATP [36].

Le couplage de la phosphorylation oxydative (c'està-dire la quantité d'oxygène nécessaire aux mitochondries pour synthétiser une quantité donnée d'ATP, ou en d'autres termes, le rendement énergétique des mitochondries) semble diminué lors du vieillissement $[42,43]$. Cette altération a récemment été vérifiée dans notre laboratoire. En utilisant la technique des fibres perméabilisées ${ }^{2}$, appliquée à une population d'hommes âgés actifs, un découplage modéré de la phosphorylation a en effet pu être observé [23]. Parce qu'un découplage modéré de la phosphorylation oxydative résulte généralement en une diminution de la production d'EA0, il pourrait représenter un mécanisme compensatoire visant à limiter la production mitochondriale d'EAO [42, 44]. Cette hypothèse reste cependant à être confirmée.

L'énergétique mitochondriale semble donc altérée au cours du vieillissement. Cette altération de l'énergétique mitochondriale représente un mécanisme qui contribue très probablement au déclin fonctionnel des muscles squelettiques survenant lors du vieillissement.

\section{Capacité mitochondriale de rétention en calcium, apoptose et vieillissement}

Les mitochondries jouent un rôle important dans l'homéostasie calcique intracellulaire. Alors qu'une augmentation modérée de la concentration en calcium dans la matrice mitochondriale a un effet positif sur le métabolisme oxydatif mitochondrial (stimulation de l'activité de multiples enzymes mitochondriales), une concentration calcique excessive peut résulter en une augmentation de la production d'EAO et un

\footnotetext{
${ }^{2}$ La technique de fibres perméabilisées consiste en une séparation minutieuse des fibres musculaires, suivie d'un traitement à la saponine permettant la perméabilisation spécifique du sarcolemme des fibres musculaires sans endommager les membranes des mitochondries. Cette technique permet donc d'étudier les mitochondries dans leur environnement naturel.
} 
déclenchement de l'apoptose de la cellule par l'ouverture du PTP. L'étude de la réponse des mitochondries soumises à un excès de calcium montre, chez le rat [24] et chez l'homme [23, 45], que le vieillissement musculaire s'accompagne d'une réduction de la capacité de rétention en calcium des mitochondries et d'une diminution du temps nécessaire à l'ouverture du PTP. Le vieillissement musculaire s'accompagne donc d'une sensibilisation du PTP, suggérant une propension accrue des mitochondries âgées au déclenchement de l'apoptose. La proportion de noyaux positifs pour l'endonucléase G (un facteur pro-apoptotique normalement séquestré dans les mitochondries) est 3 fois supérieure chez les hommes âgés, en comparaison à des jeunes adultes. Elle est, de plus, corrélée au temps nécessaire à l'ouverture du PTP [23]. Ces résultats, qui indiquent que le vieillissement musculaire s'accompagne d'une augmentation de l'apoptose contrôlée par les mitochondries, sont à relier aux observations qui avaient été réalisées sur des modèles animaux [17, 46]. Dans ces modèles, Chabi et al. avaient en effet rapporté que les taux de libération de cytochrome $C$ et d'endonucléase $G$ étaient entre 3, 5 et 7 fois supérieurs dans les mitochondries sous-sarcolemmales isolées de muscle âgés, par rapport à ceux mesurés dans les mitochondries isolées d'animaux adultes jeunes. Leeuwenburgh et al. ont, quant à eux, obtenu des résultats montrant que le vieillissement musculaire chez la souris était associé à une augmentation du nombre de noyaux positifs au marquage TUNEL (un marquage détectant I'ADN clivé, marqueur d'apoptose), une augmentation de l'activité de la caspase 3 (une caspase activée par la sortie du cytochrome $c$ de l'espace inter-membranaire), et une translocation de l'endonucléase G, au niveau nucléaire [46].

Ces résultats indiquent donc clairement que l'apoptose contrôlée par les mitochondries augmente lors du vieillissement musculaire. Celle-ci pourrait être à l'origine du mécanisme contribuant aux pertes de masse et de force musculaires survenant au cours du vieillissement.

\section{Mécanismes potentiellement à l'origine de l'accumulation de dysfonctions mitochondriales au cours du vieillissement musculaire}

L'implication d'une surproduction mitochondriale d'EAO comme mécanisme responsable du vieillissement musculaire, un pilier de la théorie de Harman, est actuellement sujette à controverse. Bien qu'il soit prématuré d'écarter cette hypothèse, cette controverse suggère que d'autres mécanismes cellulaires pourraient être impliqués dans l'accumulation de mitochondries dysfonctionnelles au cours du vieillissement musculaire. Des études récentes proposent que des altérations de la mitophagie (le processus responsable de I'élimination des mitochondries dysfonctionnelles $[47,48])(\rightarrow)$ pourraient être impliquées dans le développement de la sarcopénie.

Des résultats indiquant des défauts de la mito-

$(\rightarrow)$ Voir la Synthèse de P. Vigié et $\mathrm{N}$. Camougrand, $m / s n^{\circ} 3$, mars 2017, page 231 phagie lors du vieillissement musculaire ont en effet été rapportés chez I'homme [23, 49]. Plus spécifiquement, ces observations suggèrent que l'axe de régulation de la mitophagie Parkin-PINKI ${ }^{3}$ pourrait être altéré au cours du vieillissement musculaire. En effet, le ratio Parkin/VDAC (canal à anions dépendant du voltage facilitant le recrutement de Parkin sur les mitochondries dysfonctionnelles, étape nécessaire pour l'initiation de la mitophagie) apparaît être réduit dans les muscles des hommes âgés [23]. Le contenu en Parkin est également réduit dans les muscles des femmes âgées frêles [49]. II semble donc qu'il existe effectivement une altération globale des mécanismes autophagiques au cours du vieillissement musculaire [14, 50,51]. Dans une étude récente, Rana et ses collaborateurs ont montré, chez la drosophile, que la surexpression de Parkin entraînait une augmentation de la longévité de l'insecte, qui était associée à un accroissement de l'activité de la citrate synthase et une réduction des agrégats protéiques dans les muscles des insectes âgés [52]. L'ensemble de ces travaux suggèrent ainsi que le potentiel mitophagique pourrait être réduit lors du vieillissement musculaire. Cette altération représenterait donc un mécanisme cellulaire séduisant qui pourrait expliquer l'accumulation de mitochondries dysfonctionnelles au cours du vieillissement musculaire. Un autre mécanisme qui pourrait également contribuer à l'accumulation de dysfonctions mitochondriales au cours du vieillissement musculaire repose sur une altération des processus contrôlant la dynamique mitochondriale. Alors que les mitochondries ont, pendant très longtemps, été considérées comme des organites à la structure figée (en forme de petit pois ou de petite sphère), il est maintenant bien établi qu'elles peuvent arborer une architecture complexe dans la plupart des types cellulaires, particulièrement dans les cellules musculaires [53]. Les mitochondries forment en effet un réseau dynamique [53] dont les composants sont capables de changer de morphologie par des processus de fusion (régulés par les protéines pro-fusion mitofusin 1 et $2[\mathrm{Mfn}-1,-2]$ et optic atrophy 1 [OPAl]), et de fission (régulés par les protéines pro-fission dynamin-related protein 1 [DRPl] et mitochondrial-fission 1 protein [FISI]) [53]. Au fur et à mesure que la machinerie moléculaire qui contrôle cette dynamique et cette morphologie a été révélée, il est apparu évident que morphologie et fonctions des mitochondries étaient interdépendantes [53]. Bien que les relations exactes entre morphologie et fonctions des mitochondries restent à établir, il est désormais admis que les modifications de la première affectent généralement les secondes et vice-versa [53]. Notre groupe a mis en évidence que

${ }_{3}^{3}$ Parkin est une ubiquitine ligase jouant un rôle clé dans la régulation de la mitophagie ; PINKI (PTEN-induced putative kinase 1) est une protéine recrutant Parkin sur les mitochondries endommagées. 

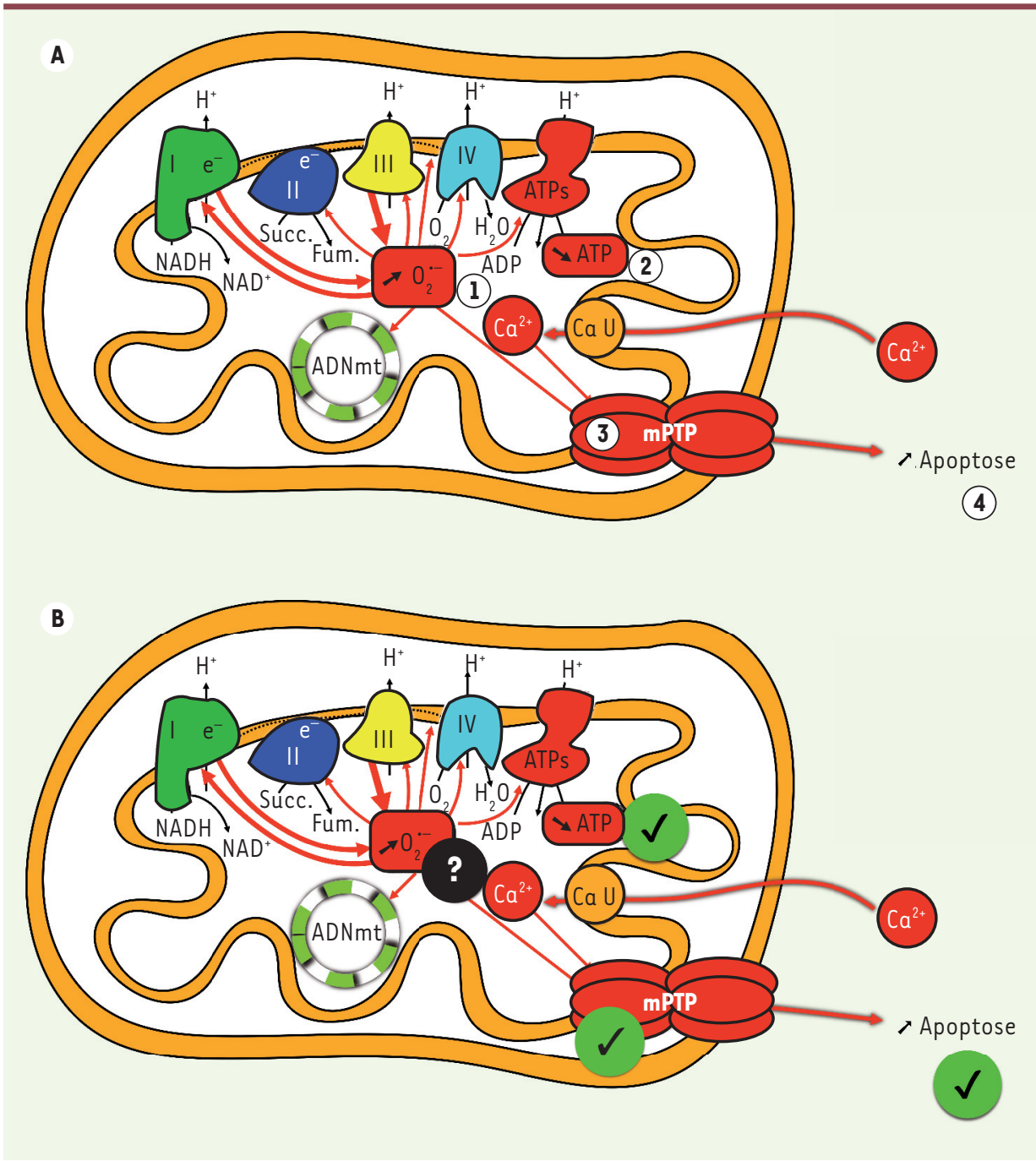

Figure 3. Dysfonctions mitochondriales et vieillissement musculaire. A. Les 4 piliers de la théorie mitochondriale du vieillissement musculaire: (1) ) surproduction d'anion superoxyde $\left(\mathrm{O}_{2}{ }^{--}\right.$, un radical libre dérivé de l'oxygène) causant des dommages aux différentes macromolécules des mitochondries. (2) Altération, avec le vieillissement, de l'énergétique mitochondriale (c'està-dire de la production d'ATP). (3) Sensibilisation du pore de transition de perméabilité (PTP) et (4) déclenchement de l'apoptose. B. État des connaissances quant aux différents piliers de la théorie mitochondriale du vieillissement. Les cercles verts cochés identifient les piliers pour lesquels un consensus existe dans la littérature. Les cercles contenant un point d'interrogation illustrent les piliers controversés. I, II, III, IV : complexes protéiques de la chaîne respiratoire; I: complexe I (NADH-coenzyme $\rho$ oxydoréductase) ; II : complexe II (succinate-coenzyme $\rho$ oxydoréductase); III : complexe III (coenzyme (-cytochrome ( oxydoréductase);

IV : complexe IV (cytochrome c oxydase) ; ATPs : ATP synthase ; mPTP : pore de transition de perméabilité mitochondrial ; ADNmt : ADN mitochondrial ; $\mathrm{Ca} \mathrm{U}$ : unipore calcique ; $\mathrm{O}_{2}{ }^{-\cdot}$ : anion super oxyde ; Fum : fumarate ; NAD : nicotinamide adénine dinucléotide ; NADH : nicotinamide adénine dinucléotide réduit; Succ : succinate.

morphologie et dynamique des mitochondries étaient altérées au cours du vieillissement [54]. Chez la souris, nous avons mis en évidence que le vieillissement musculaire était associé à une augmentation de la complexité des mitochondries intermyofibrillaires ${ }^{4}$ et à un élargissement des mitochondries sous-sarcolemmales ${ }^{5}$ [54]. En lien avec ces résultats, il a également été rapporté que le ratio $\mathrm{Mfn}-2 / \mathrm{DRPl}$ était augmenté chez les souris âgées, suggérant une augmentation du processus de fusion mitochondriale au cours du vieillissement musculaire [54].

Une morphologie trop complexe peut potentiellement produire des effets néfastes sur le fonctionnement des mitochondries. En effet, l'inhibition spécifique de Drpl a pour résultat une hyperfusion des mitochondries qui est associée à une diminution de la synthèse d'ATP mitochondriale [55].

\footnotetext{
${ }^{4}$ Les mitochondries intermyofibrillaires sont localisées entre les myofibrilles.

${ }^{5}$ Les mitochondries sous-sarcolemmales sont localisées juste sous la membrane des cellules musculaires.
}

Ainsi, les altérations de la dynamique et de la morphologie des mitochondries, rapportées par Leduc-Gaudet et al., dans les muscles âgés, pourraient représenter un mécanisme contribuant à l'accumulation de dysfonctions mitochondriales au cours du vieillissement et, ainsi, au développement de la sarcopénie [54].

\section{Conclusions}

Il apparaît donc clairement que le vieillissement musculaire s'accompagne d'une accumulation de dysfonctions mitochondriales, incluant une altération de l'énergétique mitochondriale, une moindre capacité de rétention calcique, une sensibilisation du PTP, et un déclenchement de l'apoptose. Alors que le, ou les, 
mécanismes impliqués restent, à l'heure actuelle, encore incompris, des études récentes suggèrent qu'une réduction du potentiel mitophagique et qu'une altération de la dynamique et de la morphologie des mitochondries pourraient être impliquées dans cette accumulation de dysfonctions au cours du vieillissement. L'étude des rôles joués par les processus régulant la mitophagie et la dynamique des mitochondries dans le développement de la sarcopénie représente donc des perspectives de recherche extrêmement intéressantes qui pourraient conduire au développement de nouvelles stratégies thérapeutiques afin de lutter contre la sarcopénie. $\diamond$

\section{SUMMARY}

Skeletal muscle aging and mitochondrial dysfunction: an update One of the most obvious and deleterious changes occurring with aging is a progressive loss of skeletal muscle mass and strength, a physiological process named sarcopenia. Amongst the multiple theories that have been put forward to explain sarcopenia, the mitochondrial theory of aging, which postulates that the accumulation of mitochondrial dysfunctions with aging plays a causal role in muscle atrophy, has focused intense research effort and attention in the past decades. The generally accepted view of this theory is that, due to the reactive oxygen species (ROS) production inherent to respiratory chain activity, oxidative damage to mitochondrial proteins, lipids and DNA accumulates with aging. This damage is thought to (i) exacerbate mitochondrial ROS production, (ii) impair the capacity of mitochondria to adequately match the cellular ATP demand and (iii) trigger mitochondrial-mediated apoptosis. Although very appealing, this theory remains controversial. The aims of the present review are (i) to provide the reader with a short, but comprehensive review of the current literature linking mitochondrial dysfunction and sarcopenia and (ii) to briefly discuss the potential mechanisms underlying the accumulation of mitochondrial dysfunction with muscle aging. $\diamond$

\section{LIENS D’INTÉRÊT}

Les auteurs déclarent n'avoir aucun lien d'intérêt concernant les données publiées dans cet article.

\section{RÉFÉRENCES}

1. Cruz-Jentoft AJ, Baeyens JP, Bauer JM, et al. Sarcopenia: European consensus on definition and diagnosis: Report of the European Working Group on Sarcopenia in Older People. Age Ageing 2010 ; $39: 412-23$.

2. Janssen I, Shepard DS, Katzmarzyk PT, Roubenoff R. The healthcare costs of sarcopenia in the United States. J Am Geriatr Soc $2004 ; 52: 80-5$.

3. Gouspillou G, Picard M, Godin R, et al. Role of peroxisome proliferator-activated receptor gamma coactivator 1-alpha (PGC-lalpha) in denervation-induced atrophy in aged muscle: facts and hypotheses. Longev Healthspan $2013 ; 2: 13$.

4. Friedman JR, Nunnari J. Mitochondrial form and function. Nature $2014 ; 505: 335-43$.

5. Sarewicz M, Osyczka A. Electronic connection between the quinone and cytochrome $C$ redox pools and its role in regulation of mitochondrial electron transport and redox signaling. Physiol Rev $2015 ; 95: 219-43$.

6. Elustondo PA, Nichols M, Robertson GS, Pavlov EV. Mitochondrial $\mathrm{Ca}^{2+}$ uptake pathways. J Bioenerg Biomembr 2017 ; 49 : 113-9.

7. Halestrap AP. What is the mitochondrial permeability transition pore? J Mol Cell Cardiol $2009 ; 46$ : 821-31.

8. Richardson AP, Halestrap AP. Quantification of active mitochondrial permeability transition pores using GNX-4975 inhibitor titrations provides insights into molecular identity. Biochem J 2016 ; $473: 1129-40$.

9. Giorgio V, von Stockum S, Antoniel M, et al. Dimers of mitochondrial ATP synthase form the permeability transition pore. Proc Natl Acad Sci USA 2013 ; 110 : 5887-92.
10. Dupont-Versteegden દદ. Apoptosis in skeletal muscle and its relevance to atrophy. World J Gastroenterol 2006 ; 12 : 7463-6.

11. Harman D. Aging: a theory based on free radical and radiation chemistry.J Gerontol 1956; $11: 298-300$.

12. Harman D. The biologic clock: the mitochondria? J Am Geriatr Soc $1972 ; 20$ : 145-7.

13. Sinha-Hikim I, Sinha-Hikim AP, Parveen M, et al. Long-term supplementation with a cystine-based antioxidant delays loss of muscle mass in aging. J Gerontol A Biol Sci Med Sci 2013 ; 68 : 749-59.

14. Wohlgemuth SE, Seo AY, Marzetti $\varepsilon$, et al. Skeletal muscle autophagy and apoptosis during aging: effects of calorie restriction and life-long exercise. Exp Gerontol 2010; 45 : 138-48.

15. Kovacheva EL, Sinha Hikim AP, Shen R, et al. Testosterone supplementation reverses sarcopenia in aging through regulation of myostatin, c-Jun $\mathrm{NH}_{2}$ terminal kinase, Notch, and Akt signaling pathways. Endocrinology 2010 ; $151: 628-38$.

16. Yarian CS, Rebrin I, Sohal RS. Aconitase and ATP synthase are targets of malondialdehyde modification and undergo an age-related decrease in activity in mouse heart mitochondria. Biochem Biophys Res Commun 2005; 330 : 151-6.

17. Chabi B, Ljubicic V, Menzies KJ, et al. Mitochondrial function and apoptotic susceptibility in aging skeletal muscle. Aging Cell $2008 ; 7: 2-12$.

18. Capel F, Rimbert V, Lioger D, et al. Due to reverse electron transfer, mitochondrial $\mathrm{H} 202$ release increases with age in human vastus lateralis muscle although oxidative capacity is preserved. Mech Ageing Dev 2005 ; $126: 505-11$.

19. Dirks AJ, Hofer T, Marzetti $\varepsilon$, et al. Mitochondrial DNA mutations, energy metabolism and apoptosis in aging muscle. Ageing Res Rev 2006 ; 5 : 179-95.

20. Lanza IR, Zabielski P, Klaus KA, et al. Chronic caloric restriction preserves mitochondrial function in senescence without increasing mitochondrial biogenesis. Cell Metab $2012 ; 16: 777-88$.

21. Umanskaya A, Santulli G, Xie W, et al. Genetically enhancing mitochondrial antioxidant activity improves muscle function in aging. Proc Natl Acad Sci USA 2014 ; 111 : 15250-5.

22. Hütter $\varepsilon$, Skovbro $M$, Lener $B$, et al. Oxidative stress and mitochondrial impairment can be separated from lipofuscin accumulation in aged human skeletal muscle. Aging Cell $2007 ; 6: 245-56$.

23. Gouspillou, Sgarioto N, Kapchinsky S, et al. Increased sensitivity to mitochondrial permeability transition and myonuclear translocation of endonuclease $\mathrm{G}$ in atrophied muscle of physically active older humans. FASEB J $2014 ; 28: 1621-33$.

24. Picard M, Ritchie D, Wright KJ, et al. Mitochondrial functional impairment with aging is exaggerated in isolated mitochondria compared to permeabilized myofibers. Aging Cell $2010 ; 9$ : 1032-46.

25. Sakellariou GK, Pearson T, Lightfoot AP, et al. Long-term administration of the mitochondria-targeted antioxidant mitoquinone mesylate fails to attenuate age-related oxidative damage or rescue the loss of muscle mass and function associated with aging of skeletal muscle. FASEB J 2016; 30 : 3771-85.

26. Hou Y, Li S, Wu M, et al. Mitochondria-targeted peptide SS-31 attenuates renal injury via an antioxidant effect in diabetic nephropathy. Am J Physiol Renal Physiol 2016 ; 310 : F547-59.

27. Capel F, Buffière C, Mirand PP, Mosoni L. Differential variation of mitochondrial $\mathrm{H}_{2} \mathrm{O}_{2}$ release during aging in oxidative and glycolytic muscles in rats. Mech Ageing Dev 2004 ; 125 : 367-73.

28. Rasmussen UF, Krustrup P, Kjaer M, Rasmussen HN. Human skeletal muscle mitochondrial metabolism in youth and senescence: no signs of functional changes in ATP formation and mitochondrial oxidative capacity. Pflüg Arch $2003 ; 446: 270-8$.

29. Tonkonogi M, Fernström M, Walsh B, et al. Reduced oxidative power but unchanged antioxidative capacity in skeletal muscle from aged humans. Pflüg Arch $2003 ; 446: 261-9$.

30. Trounce I, Byrne $\varepsilon$, Marzuki S. Decline in skeletal muscle mitochondrial respiratory chain function: possible factor in ageing. Lancet $1989 ; 333: 637-9$.

31. Conley KE, Jubrias SA, Esselman PC. Oxidative capacity and ageing in human muscle. J Physiol 2000 ; $526: 203-10$.

32. Picard M, Ritchie $D$, Thomas MM, et al. Alterations in intrinsic mitochondrial function with aging are fiber type specific and do not explain differential atrophy between muscles. Aging Cell 2011; 10 : 1047-55.

33. Kumaran S, Panneerselvam KS, Shila S, et al. Age-associated deficit of mitochondrial oxidative phosphorylation in skeletal muscle: role of carnitine and lipoic acid. Mol Cell Biochem $2005 ; 280: 83-9$. 


\section{RÉFÉRENCES}

34. Zangarelli A, Chanseaume $\varepsilon$, Morio B, et al. Synergistic effects of caloric restriction with maintained protein intake on skeletal muscle performance in 21-month-old rats: a mitochondria-mediated pathway. FASEB J $2006 ; 20: 2439-50$

35. Gouspillou, Bourdel-Marchasson I, Rouland R, et al. Alteration of mitochondrial oxidative phosphorylation in aged skeletal muscle involves modification of adenine nucleotide translocator. Biochim Biophys Acta $2010 ; 1797$ : 143-51.

36. Gouspillou, Bourdel Marchasson I, Rouland R, et al. Mitochondrial energetics is impaired in vivo in aged skeletal muscle. Aging Cell $2014 ; 13: 39-48$.

37. Figueiredo PA, Ferreira RM, Appell HJ, Duarte JA. Age-induced morphological, biochemical, and functional alterations in isolated mitochondria from murine skeletal muscle. J Gerontol A Biol Sci Med Sci $2008 ; 63: 350-9$

38. Short KR, Bigelow ML, Kahl J, et al. Decline in skeletal muscle mitochondrial function with aging in humans. Proc Natl Acad Sci USA 2005 ; 102 : 5618-23.

39. Drew B, Phaneuf S, Dirks A, et al. Effects of aging and caloric restriction on mitochondrial energy production in gastrocnemius muscle and heart. Am J Physiol Regul Integr Comp Physiol 2003 $284:$ R474-80.

40. Scarabelli CC, McCauley RB, Yuan Z, et al. Oral administration of amino acidic supplements improves protein and energy profiles in skeletal muscle of aged rats: elongation of functional performance and acceleration of mitochondrial recovery in adenosine triphosphate after exhaustive exertion. Am J Cardiol 2008 ; 101 : S42-8.

41. Mansouri A, Muller FL, Liu Y, et al. Alterations in mitochondrial function, hydrogen peroxide release and oxidative damage in mouse hind-limb skeletal muscle during aging. Mech Ageing Dev $2006 ; 127: 298-306$

42. Amara $C \varepsilon$, Shankland $\varepsilon G$, Jubrias $S A$, et al. Mild mitochondrial uncoupling impacts cellular aging in human muscles in vivo. Proc Natl Acad Sci USA 2007; 104 : 1057-62.

43. Marcinek DJ, Schenkman KA, Ciesielski WA, et al. Reduced mitochondrial coupling in vivo alters cellular energetics in aged mouse skeletal muscle.J Physiol 2005 ; 569 : 467-73.

44. Brand MD. Uncoupling to survive? The role of mitochondrial inefficiency in ageing. Exp Gerontol 2000; $35: 811-20$.

45. Spendiff S, Vuda M, Gouspillou G, et al. Denervation drives mitochondrial dysfunction in skeletal muscle of octogenarians. J Physiol 2016 ; 594 : 7361-79.
46. Leeuwenburgh C, Gurley CM, Strotman BA, Dupont-Versteegden દદ. Agerelated differences in apoptosis with disuse atrophy in soleus muscle. Am J Physiol Regul Integr Comp Physiol 2005 ; 288 : R1288-96.

47. Youle RJ, Narendra DP. Mechanisms of mitophagy. Nat Rev Mol Cell Biol $2011 ; 12: 9-14$

48. Vigié $\mathrm{P}$, Camougrand $\mathrm{N}$. Mitophagie et contrôle qualité des mitochondries. Med Sci (Paris) $2017 ; 33: 231-7$.

49. Drummond MJ, Addison 0 , Brunker L, et al. Downregulation of $\varepsilon 3$ ubiquitin ligases and mitophagy-related genes in skeletal muscle of physically inactive, frail older women: a cross-sectional comparison. J Gerontol A Biol Sci Med Sci $2014 ; 69$ : 1040-8.

50. O'Leary MF, Vainshtein A, lqbal S, et al. Adaptive plasticity of autophagic proteins to denervation in aging skeletal muscle. Am J Physiol Cell Physiol $2013 ; 304$ : C422-30

51. Carnio S, LoVerso F, Baraibar MA, et al. Autophagy impairment in muscle induces neuromuscular junction degeneration and precocious aging. Cell Rep 2014 ; 8 : 1509-21.

52. Rana $A$, Rera M, Walker DW. Parkin overexpression during aging reduces proteotoxicity, alters mitochondrial dynamics, and extends lifespan. Proc Natl Acad Sci USA 2013 ; 110 : 8638-43.

53. Picard M, Taivassalo T, Gouspillou G, Hepple RT. Mitochondria: isolation, structure and function. J Physiol $2011 ; 589: 4413-21$.

54. Leduc-Gaudet JP, Picard M, St-Jean Pelletier F, et al. Mitochondria morphology is altered in atrophied skeletal muscle of aged mice. Oncotarget $2015 ; 6: 17923-37$.

55. Benard G, Bellance N, James D, et al. Mitochondrial bioenergetics and structural network organization. J Cell Sci 2007 ; 120 : 838-48.

56. Barouki R. Stress oxydant et vieillissement. Med Sci (Paris) 2006; 22 : 266-72.

TIRÉS À PART

G. Gouspillou
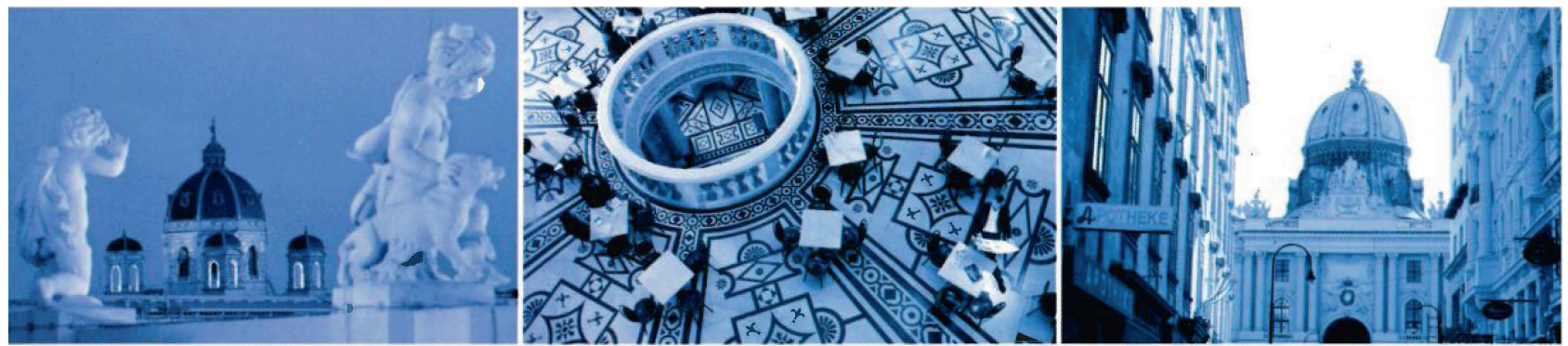
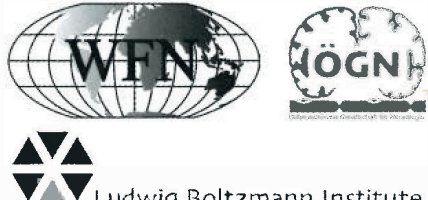

udwig Boltzmann Institute Experimental and Clinical Traumatology 